\title{
Selective transport of SC1 mRNA, encoding a putative extracellular matrix glycoprotein, during postnatal development of the rat cerebellum and retina
}

\author{
Andrea J. Mothe, Ian R. Brown * \\ Department of Zoology, University of Toronto, Scarborough Campus, 1265 Military Trail, Toronto, Ontario, Canada M1C 1A4
}

Accepted 23 November 1999

\begin{abstract}
The selective transport of mRNA species into peripheral processes of cells is an important aspect of gene expression in the nervous system. In this study, we report the transport of SC1 mRNA into the distal processes of Bergmann glial (BG) cells at particular stages of development. SC1 is a putative anti-adhesive extracellular matrix (ECM) glycoprotein that is expressed not only in the developing central nervous system (CNS) but also in the adult brain. The intracellular distribution of SC1 mRNA was examined in two highly laminated neural structures, the cerebellum and retina, during postnatal development and in the adult rat. Our results indicate that SC1 mRNA expression is both spatially and temporally regulated. SC1 message was localized to BG cell bodies at postnatal day 5 (P5) and P10. However, by P15 through to the adult, SC1 mRNA was transported to distal processes of BG cells in the synapse-rich molecular layer (ML) of the cerebellum. In the developing rat retina, SC1 mRNA was expressed in specific neuronal populations by P10, however, transport of SC1 message to the dendrites of these retinal neurons was not detected during development or in the adult. These results indicate neural mechanisms which control the timing and cell type in which selective transport of SC1 mRNA is observed. The localization of SC1 mRNA to the distal processes of BG cells in the synapse-rich ML of the cerebellum could facilitate local control of SC1 protein synthesis which may play roles in synapse formation during development and in synaptic plasticity in the adult. (C) 2000 Elsevier Science B.V. All rights reserved.
\end{abstract}

Keywords: Extracellular matrix; Bergmann glia; mRNA transport; In situ hybridization; Neural development

\section{Introduction}

The extracellular matrix (ECM) plays important roles in the development and maintenance of tissue integrity and function. In the central nervous system (CNS), ECM molecules participate in regulating many aspects of development, such as cell migration, process elongation, and synapse formation $[31,38]$. As the brain matures, the expression of many ECM molecules decreases. In contrast, $\mathrm{SC} 1$ is expressed not only during development but also in the adult rat CNS [16]. SC1 shares partial sequence homology with the ECM glycoprotein SPARC which possesses anti-adhesive properties and modulates the expression and interaction of a range of matrix components and growth factors [18]. SPARC is considered to be an anti-adhesive

\footnotetext{
* Corresponding author. Fax: +1-416-287-7642; e-mail: ibrown@scar.utoronto.ca
}

because it can selectively disrupt cellular contacts with the matrix and thereby affect changes in cell shape [18]. Rat SC1 may also have anti-adhesive features given that hevin, the human homolog of SC1, has been shown to possess anti-adhesive properties [12]. Previous work in our laboratory has found that $\mathrm{SC} 1$ protein is strongly expressed in the developing and adult cerebellum, specifically by Bergmann glial (BG) cells and their radial fibers which project into the synapse-rich molecular layer (ML) [21].

An aspect of gene expression which is receiving increasing attention is the selective targeting of specific mRNAs to particular subcellular domains $[11,23,36]$. While most neural mRNA species are confined to the cell body, a growing list of mRNAs are also found in the dendrites of neurons and glial cell processes. For example, mRNA encoding the high molecular weight microtubule associated protein MAP2 [10], and the $\alpha$-subunit of calcium/calmodulin-dependent protein kinase II (Cam II kinase) [7] are transported into the dendrites of neurons. 
Similarly, myelin basic protein (MBP) mRNA is localized to the processes of oligodendrocytes $[17,37]$. The transport of mRNA into cellular processes facilitates local control of the synthesis of selected proteins [35]. This gives rise to the possibility of the translation of dendritic mRNAs in response to synaptic stimulation.

The present study examines whether SC1 mRNA is transported to cellular processes during neural development. Two neural regions which show a laminar cellular organization, namely the cerebellum and the retina, were selected for analysis. The cerebellum is advantageous for an mRNA localization study since neuronal and glial cell bodies and their cellular processes are located in specific lamini. For example, the cell bodies of Purkinje neurons and BG lie in the Purkinje cellular layer (PCL), whereas their cellular processes project into the synapse-rich ML of the cerebellum. Similarly, the highly laminar structure of the retina consists of several cell types organized in discrete cell body and synapse-rich dendritic layers. Both of these tissues have easily distinguishable synaptic layers which facilitate the determination of whether SC1 message is transported into cellular processes during development. Our present studies show that, while SC1 mRNA is expressed in particular neuronal and glial cell types, transport of this message into cellular processes is observed only in BG cells in a developmentally regulated pattern. Hence, cell-specific and stage-specific mechanisms exist which control the transport of SC1 mRNA in the cellular processes of neural cells in the mammalian brain.

\section{Materials and methods}

\subsection{Riboprobe synthesis}

The SC1 subclone [16], a 295-bp HindIII-EcoR1 fragment of the rat SC1 cDNA in pGEM-3, was used to generate ${ }^{32} \mathrm{P}$-labelled riboprobes for Northern blot analysis. Linearization with EcoR1 or HindIII and subsequent in vitro transcription incorporating ${ }^{32} \mathrm{P}$-labelled UTP using SP6 and T7 polymerases produced antisense and sense SC1 riboprobes, respectively (Promega transcription kit). For in situ hybridization studies, the 295-bp HindIII$E c o$ R1 fragment of SC1 was subcloned into the vector pBluescript KS (pBS) which was used in an in vitro transcription reaction to generate digoxigenin (DIG)labelled single-stranded RNA probes as described by Boehringer Mannheim.

\subsection{Northern blot analysis}

Total RNA from brain regions, retina, and kidney was isolated from adult Wistar rats. The cerebellum and cerebral hemispheres were removed and remaining midbrain regions constituted the brain core. Aliquots of $5 \mu \mathrm{g}$ were separated on $1.5 \%$ agarose gels containing $6 \%$ formal- dehyde and blotted onto Biotrans nylon membrane. Blots were prehybridized at $60^{\circ} \mathrm{C}$ for $3 \mathrm{~h}$ in buffer containing $50 \%$ formamide, $5 \times \mathrm{SSC}, 5 \times$ Denhardt's solution $(0.1 \%$ Ficoll, $0.1 \%$ polyvinylpyrolidone, $0.1 \%$ bovine serum albumin), $50 \mathrm{mM}$ sodium phosphate (pH 6.5), 0.1\% SDS, $1 \%$ glycine, $250 \mu \mathrm{g} / \mathrm{ml}$ yeast tRNA, and $250 \mu \mathrm{g} / \mathrm{ml}$ sheared herring testes DNA. Hybridization was carried out overnight in the same buffer containing ${ }^{32} \mathrm{P}$-labelled antisense SC1 riboprobe. Blots were washed at $70^{\circ} \mathrm{C}$ in $0.1 \times$ SSC, $0.1 \%$ SDS and exposed to Kodak X-OMAT film at $-70^{\circ} \mathrm{C}$ with a Cronex Lightening Plus intensifying screen. Duplicate blots were stained with methylene blue to verify equal loading of RNA in each lane.

\subsection{Tissue preparation}

Anesthetized Wistar rats were perfused intracardially at postnatal day 1 (P1), P5, P10, P15, P20, and adult with 0.1 $M$ phosphate-buffered saline (PBS, $\mathrm{pH}$ 7.4) followed by PBS-buffered 4\% paraformaldehyde. Brains and eyes were removed and postfixed in $4 \%$ paraformaldehyde overnight at $4^{\circ} \mathrm{C}$, and then equilibrated by subsequent overnight incubations in 5\%, 10\%, and 20\% sucrose in PBS, pH 7.4. Tissue was mounted in OCT embedding compound and stored at $-70^{\circ} \mathrm{C}$.

\subsection{Non-radioactive in situ hybridization}

Cryostat tissue sections of $20 \mu \mathrm{m}$ were collected onto gelatin-coated slides ( $1 \%$ gelatin, $0.5 \%$ chromium potassium sulfate) and air-dried overnight. Brain sections were postfixed in $4 \%$ paraformaldehyde for $5 \mathrm{~min}$ and retinal sections were postfixed for $30 \mathrm{~min}$ prior to protease treatment. Concentrations in ranges of 1.0, 1.8, 2.5, and 3.5 $\mu \mathrm{g} / \mathrm{ml}$ of protease $\mathrm{K}$ were used for $\mathrm{P} 5, \mathrm{P} 10, \mathrm{P} 15$, $\mathrm{P} 20 /$ adult brain, respectively. Protease K concentrations in ranges of $0.5,1.5,2.0$, and $3.0 \mu \mathrm{g} / \mathrm{ml}$ were used for retinal stages P1, P5, P10/P15, P20/adult, respectively. A range of protease concentrations were used for each developmental stage to ensure thorough probe penetration into the tissue. Results shown are representative of optimal conditions. Incubation with protease was at $37^{\circ} \mathrm{C}$ for 20 $\mathrm{min}$ in $100 \mathrm{mM}$ Tris, $50 \mathrm{mM}$ EDTA (pH 7.5). Tissue was then washed for $10 \mathrm{~min}$ in TEA ( $\mathrm{pH} 8.0)$, and $0.1 \mathrm{M}$ PBS ( $\mathrm{pH}$ 7.4) for $15 \mathrm{~min}$ before prehybridization in a solution consisting of $50 \%$ formamide, $0.1 \mathrm{M}$ Tris $(\mathrm{pH} \mathrm{8.0)}, 5 \times$ Denhardt's, $0.1 \%$ SDS, $0.05 \% \mathrm{~N}$-lauroylsarcosine, and 50 $\mathrm{mM}$ PIPES ( $\mathrm{pH}$ 7.6). Tissue sections were hybridized overnight at $55^{\circ} \mathrm{C}$ in prehybridization buffer containing $500 \mathrm{ng} /$ section of DIG-labelled antisense or sense riboprobes. Following hybridization, slides were rinsed in TES buffer $(10 \mathrm{mM}$ Tris, $1 \mathrm{mM}$ EDTA, $500 \mathrm{mM} \mathrm{NaCl}$, pH 8.0 ), followed by a 20 -min incubation at $37^{\circ} \mathrm{C}$ in TES containing $20 \mu \mathrm{g} / \mathrm{ml}$ RNase A. Post-hybridization washes were carried out at $37^{\circ} \mathrm{C}$ and $65^{\circ} \mathrm{C}$ for $1 \mathrm{~h}$ in TES and $0.1 \times$ SSC, respectively. Slides were rinsed in PBT buffer 
(0.1 M PBS, 0.1\% Triton X-100) and incubated with $20 \%$ fetal calf serum in PBT for $2 \mathrm{~h}$ at room temperature. After blocking, slides were incubated with anti-DIG-alkaline phosphatase antibody (Boehringer Mannheim) diluted 1:800 in blocking solution overnight at $4^{\circ} \mathrm{C}$. Sections were washed three times for $30 \mathrm{~min}$ in PBT buffer, $5 \mathrm{~min}$ in AP buffer $\left(100 \mathrm{mM}\right.$ Tris, $100 \mathrm{mM} \mathrm{NaCl}, 50 \mathrm{mM} \mathrm{MgCl}_{2}$, $0.1 \%$ Tween-20) $\mathrm{pH} 9.5$, and $5 \mathrm{~min}$ in AP buffer with 5 $\mathrm{mM}$ levamisole. Slides were incubated in NBT/BCIP colour reagent in AP buffer with $5 \mathrm{mM}$ levamisole (5 $\mu \mathrm{l} / \mathrm{ml}$ NBT solution-100 mg/ml 4-nitro blue tetrazolium chloride in $70 \%$ dimethylformamide; $3.75 \mu \mathrm{l} / \mathrm{ml} \mathrm{BCIP}$ solution-50 mg/ml 5-bromo-4-chloro-3-indolyl-phosphate in dimethylformamide; Boehringer Mannheim) for 5-10 h. Slides were rinsed in Buffer A $(100 \mathrm{mM}$ Tris, $100 \mathrm{mM}$

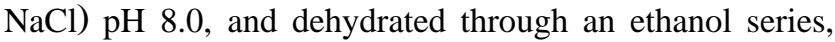
cleared in xylene, and mounted in 50\% permount $/ 50 \%$ xylene. Data representative of at least six animals per age are shown.

\subsection{Anti-glial fibrillary acidic protein (GFAP) immunohis- tochemistry}

Frozen tissue sections of $20 \mu \mathrm{m}$ were rehydrated in PBT buffer (0.1 M PBS, $0.1 \%$ bovine serum albumin, $0.2 \%$ Triton X-100) for 20 min, blocked in PBT containing $10 \%$ horse serum, and incubated overnight with anti-GFAP antibody (Boehringer Mannheim), $0.1 \mu \mathrm{g} / \mathrm{slide}$. Sections were washed in PBT and incubated for $1 \mathrm{~h}$ with biotinylated anti-mouse IgG, 1:2000. To block endogenous peroxidase activity, slides were placed in $3 \% \mathrm{H}_{2} \mathrm{O}_{2}$ for $3 \mathrm{~min}$. Sections were then washed in PBT and incubated with Vectastain reagent for $1 \mathrm{~h}$ (Vector Laboratories). Immunoreactivity was visualized by the diaminobenzidinehorseradish peroxidase reaction. Data representative of three animals per age are shown.

\section{Results}

\subsection{Northern blot analysis of SC1 mRNA in adult rat brain and retina}

Total RNA was isolated from various brain regions, retina, and kidney from the adult rat and hybridized with a ${ }^{32} \mathrm{P}$-labelled $\mathrm{SC} 1$ antisense riboprobe which recognizes a 3.2-kb mRNA [16]. As shown in Fig. 1, strong SC1 mRNA expression was observed in adult cerebellum, cerebral hemispheres and core brain regions, while only a trace signal was detected in kidney. In comparison to the brain regions, lower levels of SC1 mRNA were observed in the retina. This reflects the discrete localization of this mRNA species to specific neuronal populations in the retina as demonstrated by the in situ hybridization analysis (Figs. 5 and 6).

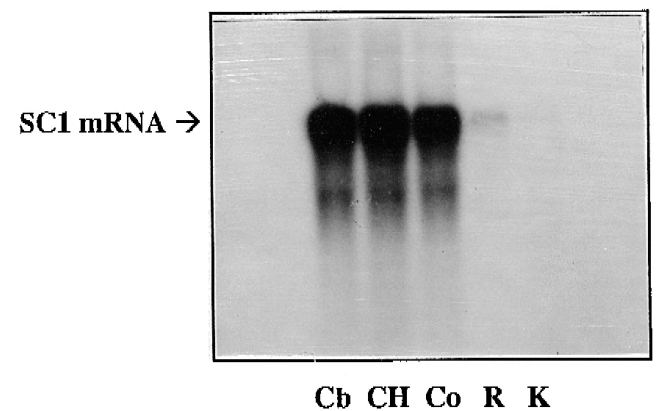

Fig. 1. Northern blot of SC1 mRNA in adult rat brain regions and retina. SC1 antisense riboprobe detected a single mRNA species of $3.2 \mathrm{~kb}$ which was highly expressed in the brain regions, and present at lower levels in retina. A trace signal was detected in adult kidney. $\mathrm{Cb}$, cerebellum; $\mathrm{CH}$, cerebral hemispheres; $\mathrm{Co}$, brain core; $\mathrm{K}$, kidney; R, retina.

\subsection{Intracellular localization of SC1 mRNA during postna- tal neural development}

The laminar organization of the cerebellum facilitates the determination of whether SC1 mRNA is transported into glial or neuronal cell processes, since cell bodies and their processes are organized in discrete layers in the cerebellum. The left panels of Fig. 2 (low magnification) indicate that SC1 mRNA was expressed in the PCL of the cerebellum. At higher magnification (right panels Fig. 2), it was apparent that this expression was localized to BG cell bodies and not to neighbouring Purkinje neurons (indicated by stars in Fig. 2). This was clearly visualized as shown in colour in Fig. 6B in which an adult cerebellar section has been hybridized with the antisense SC1 riboprobe (blue precipitate indicates mRNA signal) and counterstained with neutral red to show cellular morphology. SC1 mRNA was expressed in BG which surround the much larger cell bodies of Purkinje neurons which do not express SC1. At P5 and P10, SC1 mRNA was localized to BG cell bodies, however, transport of this mRNA was not detected in cellular processes of these glial cells at this stage in development (right panels of Fig. 2). By P15, a dramatic change in SC1 expression was evident. An increased level of SC1 mRNA expression was apparent in BG by P15 (as shown at low magnification in the left panels of Fig. 2), and this pattern was maintained at P20 and in the adult (> P60) (Fig. 6A). In addition, transport of SC1 mRNA into the radial fibers of BG cells was evident at P15 and P20 (as seen at higher magnification in the right panels of Fig. 2, arrowheads), and adult (Fig. 6B, arrowheads). The SC1 mRNA signal in these radial fibers extended from the PCL into the ML of the cerebellum (Figs. 2 and 6B). The SC1 mRNA signal was associated with BG fibers (shown in Fig. 3 by GFAP immunostaining) which are orientated as an array of parallel processes, as opposed to the highly branched dendritic tree that is characteristic of Purkinje neurons. No signal was 


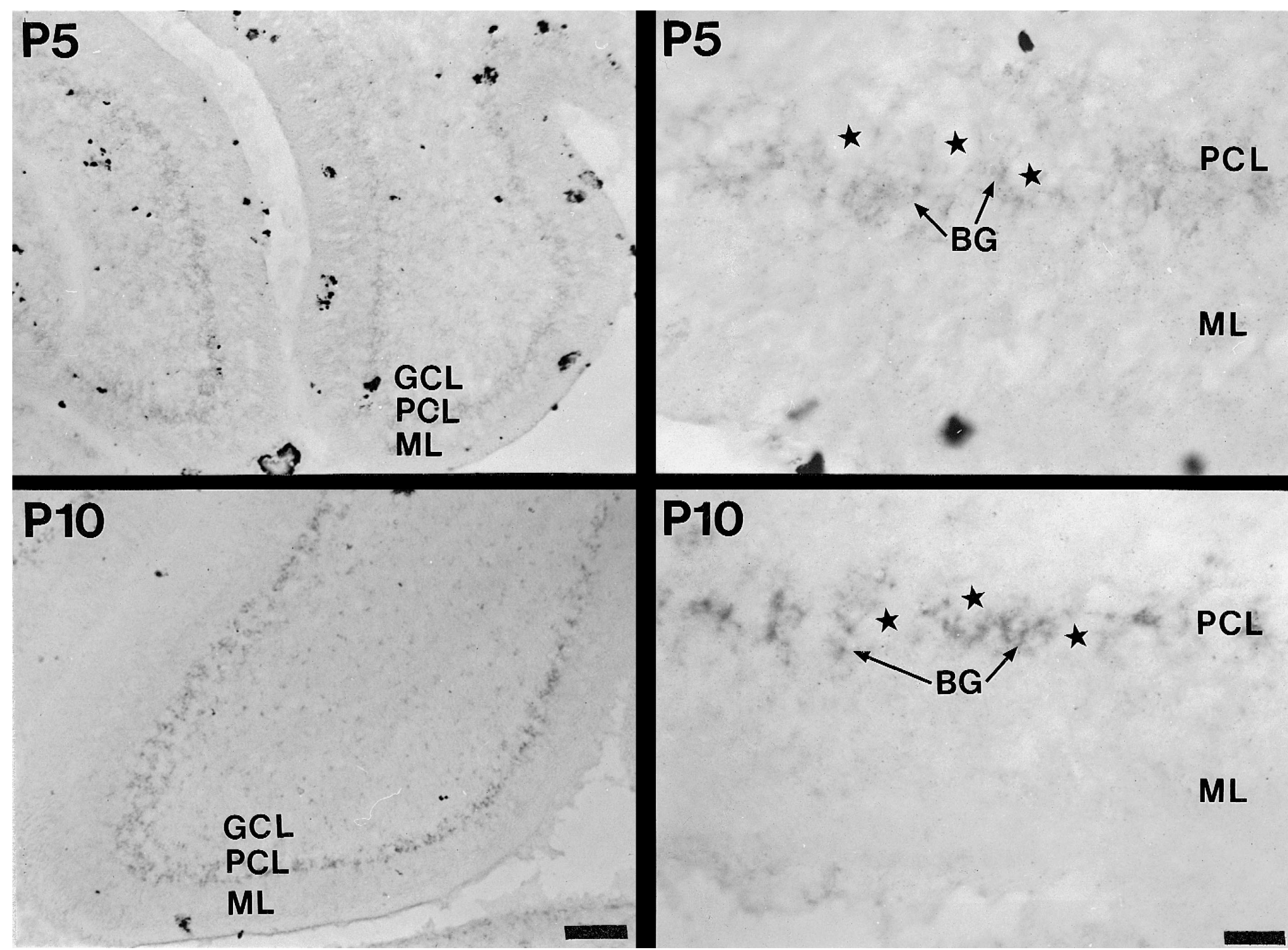

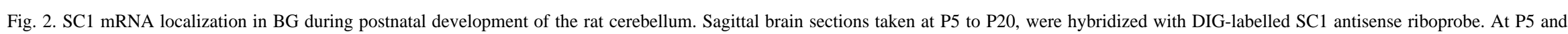

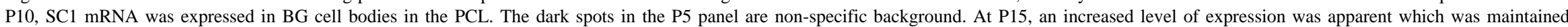

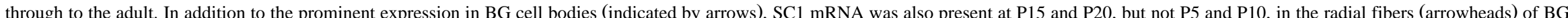

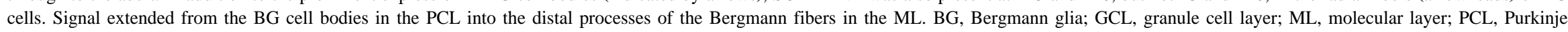

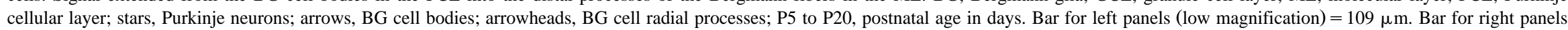
$($ high magnification $)=26.4 \mu \mathrm{m}$. 


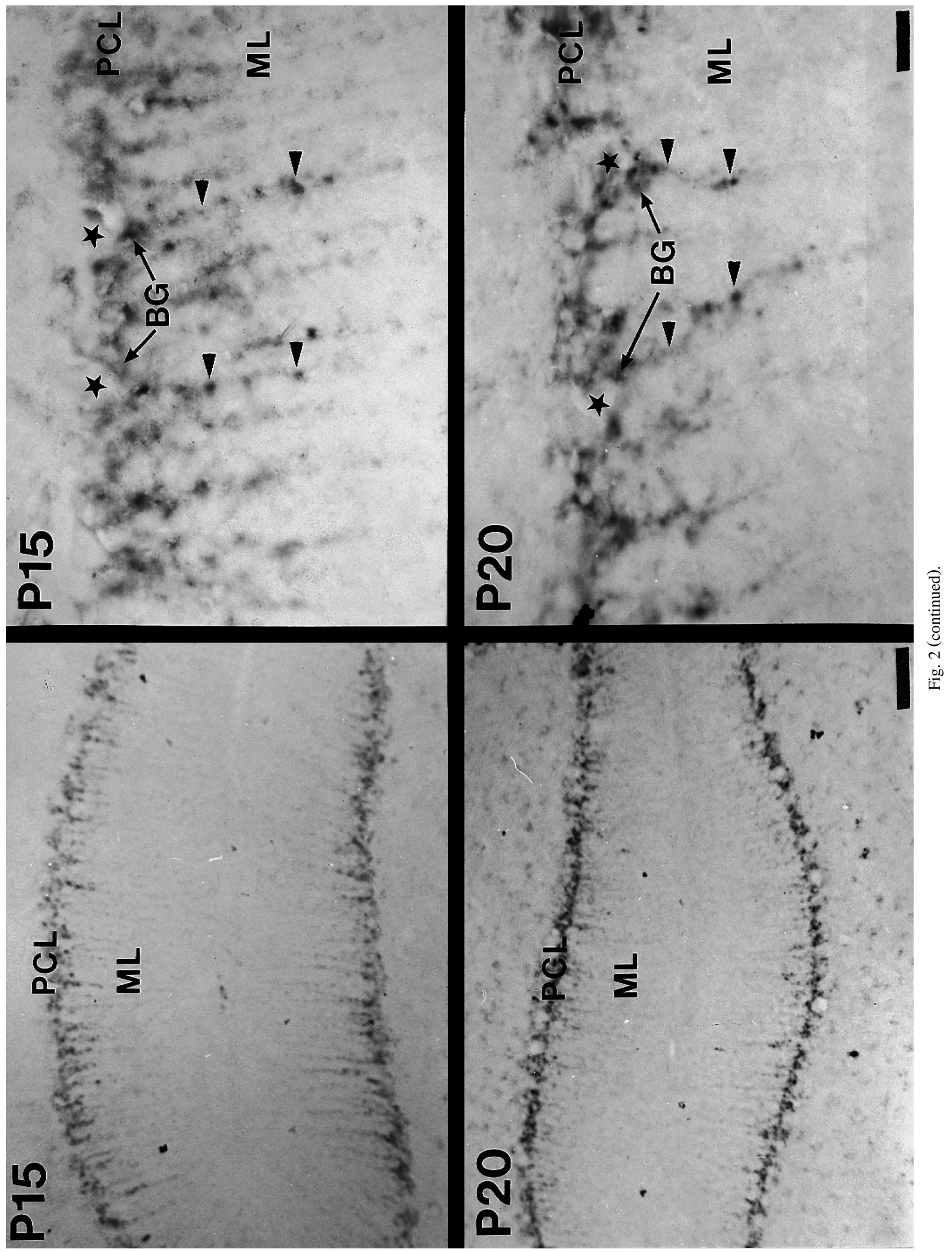




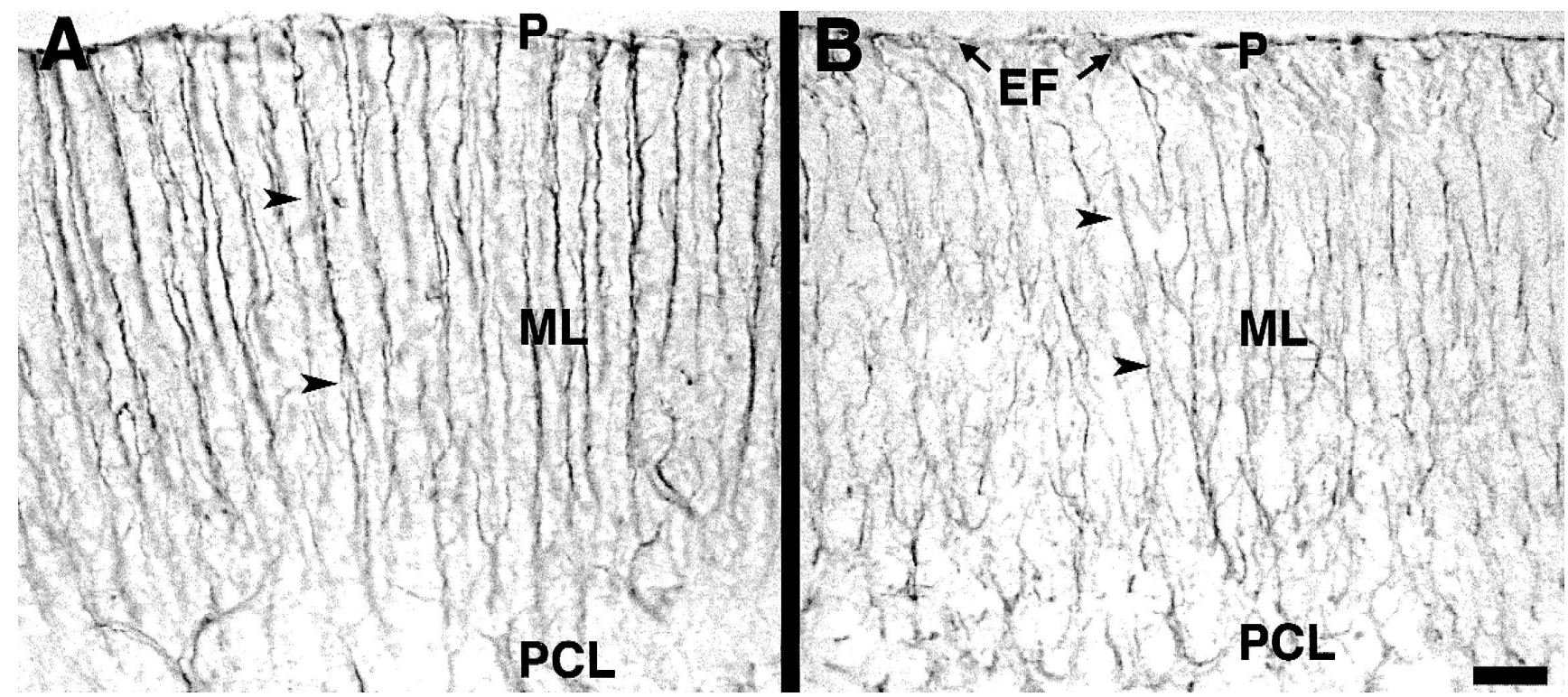

Fig. 3. GFAP immunostaining in the rat cerebellum. An antibody against GFAP was used to detect this intermediate filament protein in BG processes. Panel A: GFAP immunostaining of the BG fibers (arrowheads) traversing the ML in the adult cerebellum. Panel B: A similar pattern of GFAP staining was evident in the cerebellum at P10. As in adult, GFAP immunostaining at P10 was apparent in BG EF at the pial surface (P), and BG fibers (arrowheads) which radiate into the ML. EF, BG endfeet; ML, molecular layer; P, pia mater; PCL, Purkinje cellular layer; arrowheads, BG fibers. Bar for panels A and $\mathrm{B}=26.4 \mu \mathrm{m}$.

detected in tissue sections hybridized with a labelled sense probe (data not shown), indicating that the hybridization signal with the antisense probe was specific.

Given the lack of detection of SC1 mRNA transport at P5 and P10, it was necessary to determine whether BG fibers were present at early stages of postnatal development. Immunostaining with an antibody against the intermediate filament protein, GFAP was therefore performed on rat cerebellar sections to establish whether BG fibers span the ML at a developmental stage when SC1 message is apparent only in the cell body. Fig. 3A and B show radially orientated GFAP positive fibers (arrowheads) in the ML of the cerebellum in the adult and at P10, respectively. GFAP immunostaining was evident along BG fibers which traverse the ML to the pial surface $(\mathrm{P})$, at which point the fibers terminate as endfeet (EF) (Fig. 3). At P10, BG fibers traverse the ML, however, SC1 mRNA was not detected in these glial fibers until P15 (Fig. 2), indicating that transport of SC1 mRNA is developmentally regulated.

SC1 mRNA was strongly expressed in BG but not in adjacent Purkinje neurons in the cerebellum (Figs. 2 and 6). However, neurons of the deep cerebellar nuclei in the adult expressed SC1 mRNA which was restricted to the cell body (Fig. 4A, arrows). Neurons in the adult brain stem (Fig. 4B) and midbrain (Fig. 4C) also expressed SC1 mRNA which was apparent in the cell body (arrows) and apical dendrites (arrowheads). Transport of SC1 mRNA into distal dendritic processes of these neurons was not detected. Similar findings were also observed at P15 and P20 (data not shown). Therefore, mechanisms exist which facilitate the selective transport of SC1 mRNA into distal processes of BG cells, but not neurons, at specific stages in neural development.

\subsection{Developmental expression of SC1 mRNA in the retina}

Given the strong expression of SC1 mRNA in cerebellar BG cells and the transport of this mRNA into radial fibers of these cells, it was of interest to determine whether SC1 is similarly expressed by an analogous population of radial glia in the retina, namely the Muller cells. In addition, since SC1 mRNA was apparent in apical dendrites of brainstem and midbrain neurons, it was of interest to investigate whether $\mathrm{SC} 1 \mathrm{mRNA}$ is transported into neuronal processes in the retina. The retina is an ideal tissue for studies on mRNA localization since it is a highly ordered laminar structure composed of several neuronal populations organized in discrete cell body layers separated by synaptic-rich laminae. This laminar organization provides an ideal system to determine the intracellular distribution of mRNAs. The retina contains very few glial cell types, namely astrocytes which have migrated from the optic nerve, and Muller cells, a type of radial glia that is unique to the retina. Furthermore, the retina is particularly well suited for developmental studies on mRNA transport into cell processes since synaptogenesis occurs postnatally.

To localize SC1 mRNA at the cellular level and determine if this mRNA was transported into neural cell processes during postnatal development of the retina, a nonradioactive DIG riboprobe was employed for in situ hybridization analysis. The non-radioactive in situ results 


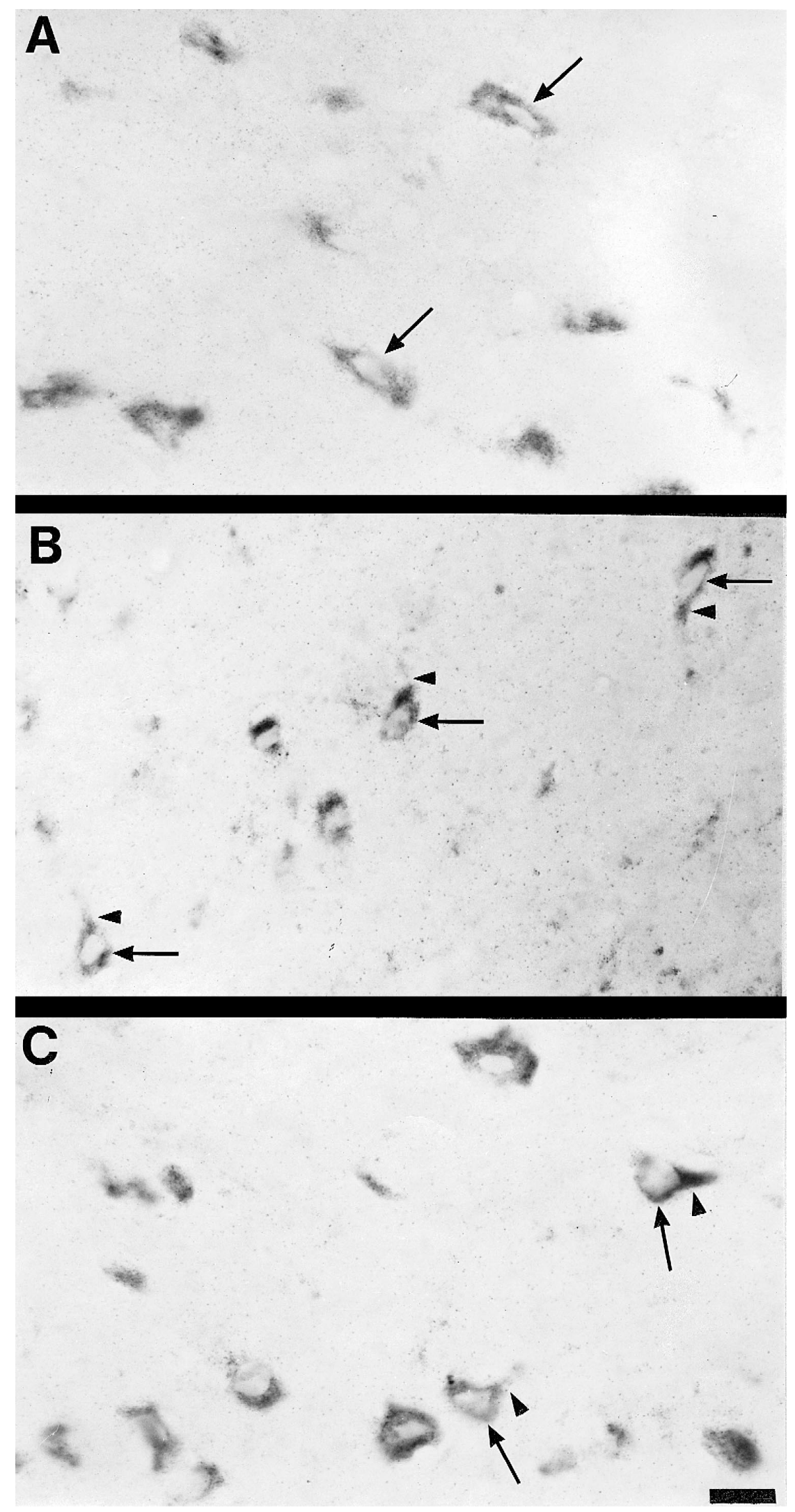

Fig. 4. Localization of SC1 mRNA in neurons of the adult rat. Panel A: SC1 mRNA was restricted to the cell body in neurons of the deep cerebellar nuclei (arrows). Panel B: Neurons in the brain stem demonstrated localization of SC1 mRNA in apical dendrites (arrowheads) in addition to a cytoplasmic localization of message (arrows). Panel C: Similarly, SC1 mRNA was evident in the cell body (arrows) and apical dendrites (arrowheads) of neurons in the midbrain. Arrows, cell body; arrowheads, apical dendrite. Bar $=26.4 \mu \mathrm{m}$. 


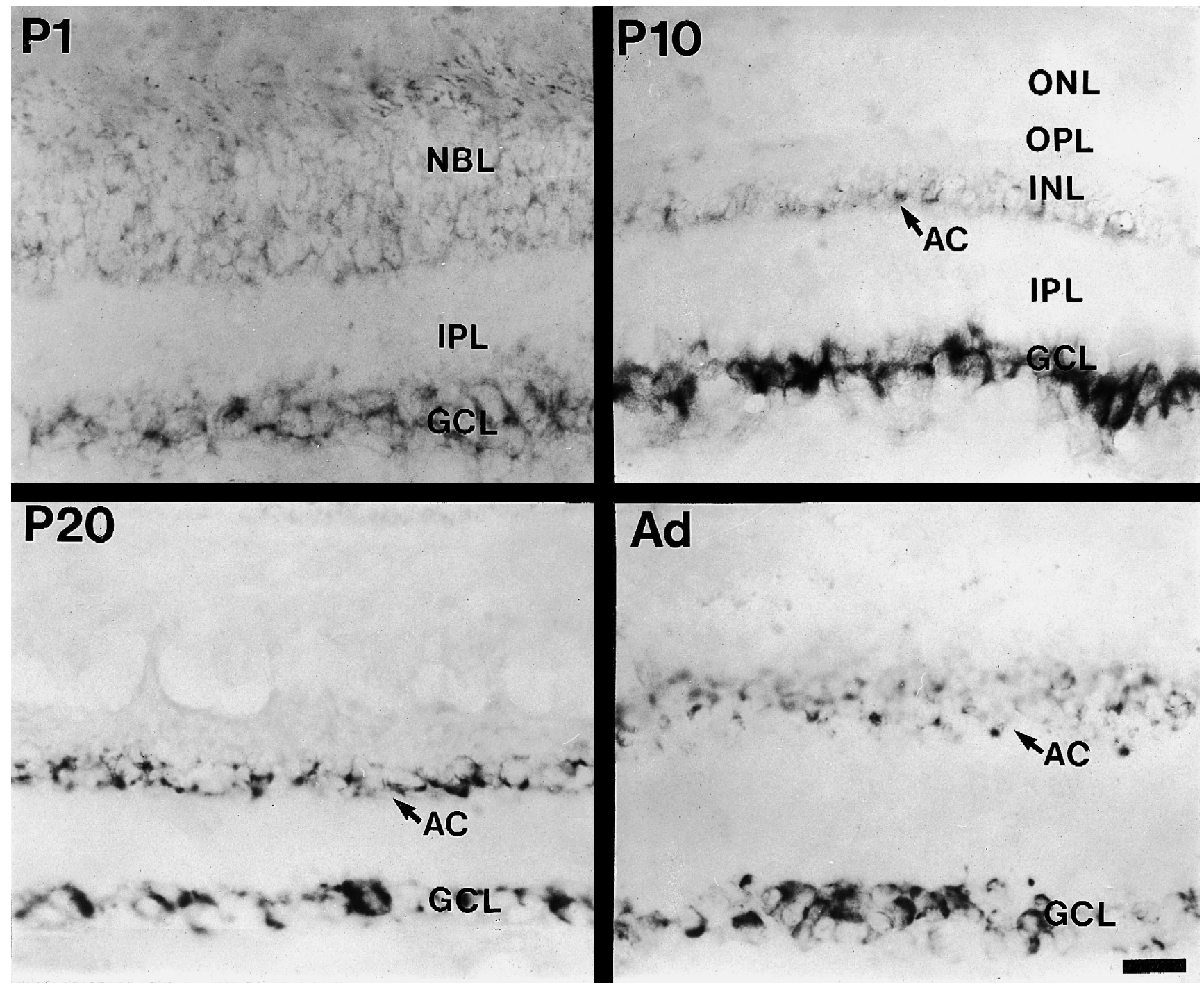

Fig. 5. Expression of SC1 mRNA in the retina during postnatal development. A non-radioactive DIG SC1 riboprobe demonstrated that SC1 mRNA was expressed in GCL and neurons of the inner portion of the INL, corresponding to the position of AC. SC1 mRNA was not detected in synaptic enriched cell processes which are concentrated in the inner and outer plexiform layers (IPL and OPL). AC, amacrine cells; GCL, ganglion cell layer; INL, inner nuclear layer; IPL, inner plexiform layer; NBL, neuroblast layer; ONL, outer nuclear layer; OPL, outer plexiform layer. Bar $=26.4 \mu \mathrm{m}$.

were confirmed by earlier studies utilizing ${ }^{35} \mathrm{~S}$-labelled riboprobes (data not shown). The DIG probes resulted in improved signal resolution since the morphology of the cell processes was more clearly distinguishable. In the newborn rat (P1), SC1 mRNA was localized to the ganglion cell layer (GCL) and a diffuse signal was also present in the inner neural retina, the neuroblast layer
(NBL) (Fig. 5). By P10, expression of SC1 mRNA segregated into two distinct bands in the GCL and inner nuclear layer (INL) reflecting localization of SC1 mRNA to ganglion and amacrine neurons (AC). As shown in the colour panels (Fig. 6C and in an adjacent counterstained section in Fig. 6D), SC1 expression in the INL was limited to the inner region where the AC are found. This pattern was

Fig. 6. SC1 mRNA visualized with DIG riboprobes in adult cerebellum and P15 retinal sections counterstained to show cellular morphology. Panel A: SC1 mRNA (blue precipitate) was localized to BG cells and their radial cellular processes in the adult cerebellum. Panel B: Higher magnification of panel A indicating the presence of SC1 mRNA in BG cells and their radial fibers (arrowheads), but not in adjacent Purkinje neurons (indicated by stars). Panels A and B were lightly counterstained with neutral red. Panel C: P15 retinal section hybridized with the non-radioactive DIG riboprobe shows high levels of SC1 mRNA in the GCL, as indicated by the dark blue signal, and lower levels in AC at the inner portion of the INL. Panel D: Adjacent P15 section was counterstained with neutral red to show that $\mathrm{SC} 1$ expression in the INL was limited to the inner region where the AC are located. AC, amacrine cells; BG, Bergmann glia; DWM, deep white matter; GCL in cerebellum, granule cell layer (panels A and B); GCL in retina, ganglion cell layer (panels C and D); INL, inner nuclear layer; IPL, inner plexiform layer; ML, molecular layer; ONL, outer nuclear layer; OPL, outer plexiform layer; PCL, Purkinje cellular layer; stars, Purkinje neurons; arrowheads, BG fibers. Bar $=109 \mu \mathrm{m}$ in panel A, $26.4 \mu \mathrm{m}$ in panels B, C and D. 


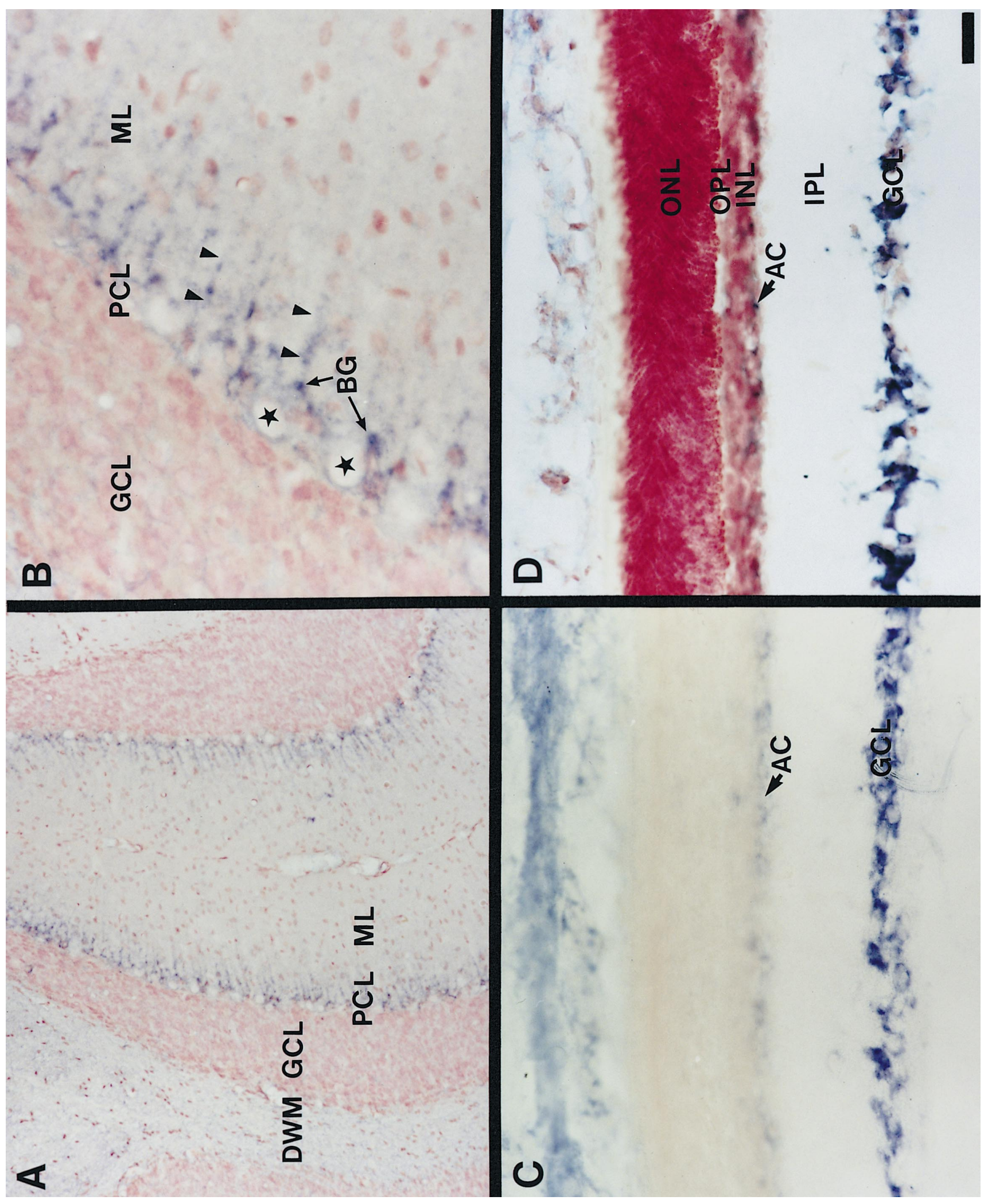


retained at P15 (Fig. 6C), P20, and in the adult (Fig. 5). There was no indication of SC1 mRNA transport into the dendrite-rich layers of the retina at any developmental stage since the inner and outer plexiform layers were consistently negative.

Interestingly, $\mathrm{SC} 1 \mathrm{mRNA}$ was not detected in retinal Muller cells. This was evident by the lack of signal in the middle portion of the INL where the Muller cell bodies are located. Furthermore, if SC1 mRNA was transported into Muller cell processes, then localization of message would be apparent in the radially orientated processes of the Muller cells which extend from the outer to the inner limiting membrane, traversing the inner and outer nuclear layer and the inner and outer plexiform layer [32,33].

\subsection{RNA transport signal (RTS) in SC1 mRNA}

The full-length SC1 cDNA sequence [16] was analyzed for the presence of sequences homologous to the 21-base RTS in the $3^{\prime}$ UTR of MBP mRNA, which has been reported to be necessary for the transport of MBP mRNA into oligodendrocyte processes [2]. As depicted in Fig. 7,
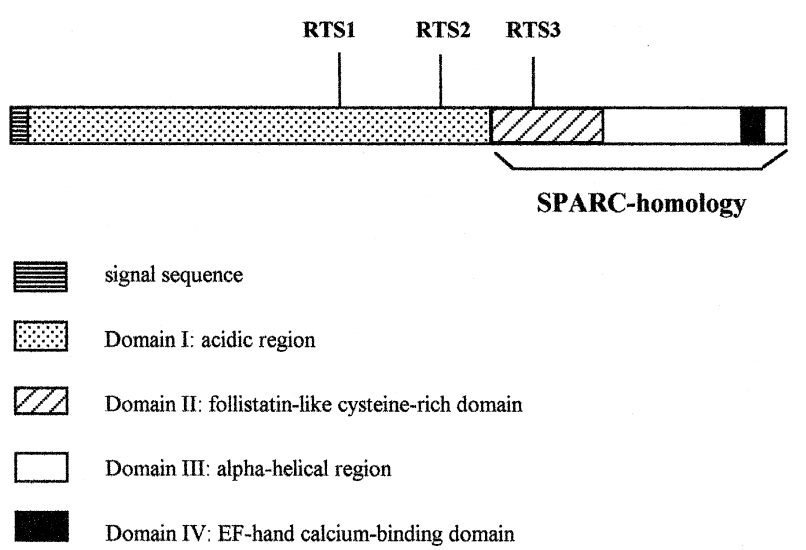
Consensus RTS
GCCAAGGAGCCAGAGAGCAUG
Rat SC1 RTS1
GCCUAGGAACCACGGAGCCAG
Rat SC1 RTS2
GCCAAGAAAGCUGAGAGCUCA
Rat SC1 RTS3
GCCAA AUCCAGAGA CGUG

Fig. 7. Schematic illustration of the modular domains in SC1 and the location of RTS motifs. SC1 has a signal sequence and the unique $\mathrm{N}$-terminal region is composed of acidic residues [16]. The carboxyl terminal region of SC1 shares a partial homology to SPARC as shown on the diagram. This region is comprised of a follistatin-like cysteine-rich domain and the EC (extracellular calcium-binding) domain, consisting of an $\alpha$-helical region and the EF-hand calcium-binding domain [20]. RTS 1 and 2 were located in the unique portion of SC1 whereas RTS 3 was found in the follistatin-like cysteine-rich domain which shares homology with SPARC. The consensus RTS was the 21-bp RNA transport sequence in the $3^{\prime}$ UTR of MBP mRNA that was shown to be necessary for the transport of MBP mRNA [2]. Bases identical to the consensus are given in non-boldface type, and blank spaces indicate missing bases in the homologous RTS.
SC1 was found to contain three putative RTS elements (indicated as RTS 1 to 3), which were located within the open reading frame. All three RTS-like elements have fourteen or more bases identical to the 21-base RTS sequence in MBP mRNA [2].

\section{Discussion}

During the development of the CNS, cell migration, process elongation, and synapse formation are some of the key events that are mediated by ECM molecules. SC1 is a putative anti-adhesive ECM glycoprotein that is expressed not only in the developing CNS but also in the adult brain, indicating that SC1 continues to play roles in the CNS after migration and proliferative events have ceased [16,22]. BG cells in the cerebellum strongly express $\mathrm{SC} 1$ and the protein is deposited along BG fibers projecting into the synapse-rich ML [21]. The present study examines the intracellular localization of SC1 mRNA in the rat cerebellum and retina during postnatal development. The selective targeting of specific mRNAs to particular intracellular domains is an important aspect of gene expression [36]. The expression of SC1 mRNA was investigated in neural regions that are highly laminated which facilitated the determination of whether SC1 mRNA is transported into neuronal or glial processes during postnatal development.

The targeting of specific mRNAs into dendrites of neurons has been studied extensively (reviewed in Refs. $[11,23])$. Only a subset of neuronal mRNAs are candidates for dendritic transport since the majority of mRNAs are localized to the cell body. Nevertheless, there is a growing list of mRNAs detected in dendrites of neurons. Certain mRNAs are also transported into processes of glial cells. In oligodendrocytes, it is well established that MBP mRNA is present in myelinating cell processes, but is confined to the cytoplasm of immature oligodendrocytes [17,37]. In addition, studies in our laboratory have demonstrated that the inducible heat shock mRNA, hsp70, is transported into oligodendrocyte processes after hyperthermia [9]. In vivo studies indicate the localization of GFAP mRNA to glial processes of reactive astrocytes and fibrous astrocytes near the glial limitans in the adult brain, and in radial glial fibers of the human fetal spinal cord [17]. The present study provides in vivo evidence of a transported mRNA which encodes a putative ECM molecule. Moreover, we are not aware of any other mRNAs that are transported in $\mathrm{BG}$, a specialized type of radial glial cell.

SC1 expression is spatially regulated since neurons in the deep cerebellar nuclei demonstrated a cytoplasmic distribution of message, while other cerebellar neurons such as granule or Purkinje cells did not express SC1. However, SC1 mRNA was strongly expressed in BG cells and selectively transported into their distal cellular processes at specific stages in development. Interestingly, SC1 
mRNA was not detected in Muller cells, a type of radial glia that is maintained in the adult retina. It has been proposed that retinal Muller cells are analogous to BG in the cerebellum, providing a scaffold over which neurons can migrate. The lack of SC1 expression in Muller cells was apparent by the absence of signal in the INL where the Muller cell bodies are situated, and by the absence of signal attributed to the characteristic radially orientated processes extending from the outer to the inner limiting membrane. In the retina, SC1 mRNA was localized to the cell bodies of ganglion and amacrine neurons by P10. However, there was no detectable transport of this message into the dendrites of these neurons at any stage in retinal development. These results suggest a spatial and temporal neural mechanism that controls the selective transport of SC1 mRNA into BG cell processes.

Targeting of mRNA into neural processes appears to depend on parameters such as developmental stage and regional location in the brain [17]. SC1 mRNA is expressed in BG cell bodies at P5 and P10 but the message is not transported into the distal processes of these cells until $\mathrm{P} 15$, a period of active synaptogenesis in the rat cerebellum. The possibility exists that a low level of SC1 mRNA is transported out at P5 and P10 but that it is below the level of detection. At P15 and thereafter, there is a very robust SC1 mRNA signal associated with BG processes. Developmental in situ studies in our laboratory have shown that calmodulin I mRNA is localized to dendrites of Purkinje neurons between P15 and P20 which also corresponds to the time of cerebellar synaptogenesis, and this mRNA is likewise restricted to cell bodies at P10 [6]. Similarly, expression of Arc mRNA increases considerably during the second and third postnatal weeks which parallels the period of morphologically defined activity-dependent restructuring of synapses [19]. The targeting of certain mRNAs to subcellular domains could allow for the rapid translation of these messages in response to synaptic stimulation [36].

During the first 3 weeks after birth, BG guide granule cell precursors from the external to the internal granule layer of the cerebellum $[3,14]$. During this period, BG fibers exhibit a number of structural changes during their differentiation. Fibers are smooth until about P12 when lateral protrusions from the radial stem processes dramatically increase in number, and at P15 resorption and differentiation of these lateral protrusions continues [8] until about P25 when the characteristic morphology of mature Bergmann fibers is attained [34]. Therefore, the differentiation of $\mathrm{BG}$ processes is dynamic involving continual growth of new processes, extension of existing ones, and retraction and resorption of others [8]. The localization of SC1 mRNA to BG processes could facilitate local synthesis of this anti-adhesive ECM molecule which may play a role in these events.

Interestingly, $\mathrm{SC} 1 \mathrm{mRNA}$ continues to be associated with processes of $\mathrm{BG}$ in the mature adult rat $(>\mathrm{P} 60)$, which implies a requirement for the selective localization of SC1 mRNA after developmental events have ceased. The transport of SC1 mRNA in distal processes of BG may be important for modulating synaptic plasticity in the adult cerebellum. Synapses in the cerebellar ML are enveloped by a BG sheath such that BG processes surround and intermingle with neuronal elements $[29,30]$. Therefore, $\mathrm{BG}$ are in an ideal position to respond to and modify events at synapses. Purkinje cell-parallel fiber synapses are tightly surrounded by BG membranes that possess a variety of receptors and ion exchangers, which could allow for glial modulation of synaptic events [24,28]. It is becoming increasingly apparent that glial cells are intimately involved in the active control of neuronal activity and synaptic transmission [5]. As has been suggested for astrocytes and Muller cells [4,25,26], BG may also play a direct role in modulating synaptic activity through changes in local calcium concentrations. Since SC1 has a calcium binding domain that is homologous to SPARC [20], SC1 could regulate local levels of calcium. Recently, it has been demonstrated that after electrical stimulation of parallel fibers in a cerebellar slice preparation, there is an increase in intracellular calcium concentration in microdomains within BG cell processes which has been suggested to interact with the particular group of synapses which they ensheath [13]. The association of SC1 mRNA with distal processes of $\mathrm{BG}$ suggests that $\mathrm{SC} 1$ protein may be locally synthesized and secreted into surrounding synaptic areas where it may act as a buffer regulating changes in local calcium concentrations.

It has been demonstrated that fluorescently tagged exogenous MBP mRNA, when microinjected into cultured oligodendrocytes, assembles into granules in the cell body and is transported into peripheral processes along microtubules [1]. Construction of chimeric MBP mRNAs have delineated the motifs that are required for mRNA transport. A 21-nucleotide sequence, termed the RNA transport signal or RTS, in the $3^{\prime}$ UTR of MBP mRNA is required for transport [2]. The RTS binds hnRNP A2, an ubiquitously expressed RNA binding protein in mammals that is most abundant in the rat brain and testis [15]. It has been suggested that the RTS may represent a cis-acting signal for RNA trafficking in a variety of different cell types given the presence of homologous sequences in several other localized mRNAs [2,27]. Three putative RTS-like elements are located within the coding region of SC1.

The results presented here indicate a spatial and temporal neural mechanism that regulates the selective transport of SC1 mRNA into BG cell processes in the cerebellum, but not into processes of retinal cells. Our report provides evidence for the transport of an mRNA that encodes an ECM protein. The selective localization of SC1 mRNA within distal processes of BG cells could facilitate local control of SC1 protein synthesis. Since SC1 is a secreted calcium-binding glycoprotein that possesses anti-adhesive properties, it may play an important role in regulating 
levels of intracellular calcium and mediating rearrangements and alterations in synaptic strengths and contacts during development and in synaptic plasticity in the adult.

\section{Acknowledgements}

We thank Sheila Rush for critical reading of this manuscript. These studies were supported by grants to I.R.B. from NSERC (Canada).

\section{References}

[1] K. Ainger, D. Avossa, F. Morgan, S.J. Hill, C. Barry, E. Barbarese, J.H. Carson, Transport and localization of exogenous myelin basic protein mRNA microinjected into oligodendrocytes, J. Cell Biol. 123 (1993) 431-441.

[2] K. Ainger, D. Avossa, A.S. Diana, C. Barry, E. Barbarese, J.H. Carson, Transport and localization elements in myelin basic protein mRNA, J. Cell Biol. 138 (1997) 1077-1087.

[3] J. Altman, Postnatal development of cerebellar cortex in the rat: maturation of the components of the granular cell layer, J. Comp. Neurol. 145 (1972) 414-465.

[4] A. Araque, R.P. Sanzgiri, V. Parpura, P.G. Haydon, Calcium elevation in astrocytes causes an NMDA receptor-dependent increase in the frequency of miniature synaptic currents in cultured hippocampal neurons, J. Neurosci. 18 (1998) 6822-6829.

[5] A. Araque, V. Parpura, R.P. Sanzgiri, P.G. Haydon, Tripartite synapses: glia, the unacknowledged partner, Trends Neurosci. 22 (1999) 208-215.

[6] F.B. Berry, I.R. Brown, CaM I mRNA is localized to apical dendrites during postnatal development of neurons in the rat brain, $\mathrm{J}$. Neurosci. Res. 43 (1996) 565-575.

[7] K.E. Burgin, M.N. Waxman, S. Rickling, S.A. Westgate, W.C. Mobley, P.T. Kelly, In situ hybridization histochemistry of $\mathrm{Ca}^{2+}$ /calmodulin-dependent protein kinase in developing rat brain, J. Neurosci. 10 (1990) 1788-1798.

[8] G.D. Das, Differentiation of Bergmann glial cells in the cerebellum: a Golgi study, Brain Res. 110 (1976) 199-213.

[9] J.A. Foster, I.R. Brown, Intracellular localization of heat shock mRNAs (hsc70 and hsp70) to neural cell bodies and processes in the control and hyperthermic rabbit brain, J. Neurosci. Res. 46 (1996) 652-665.

[10] C.C. Garner, R.P. Tucker, A. Matus, Selective localization of messenger RNA for cytoskeletal protein MAP2 in dendrites, Nature 336 (1988) 674-677.

[11] F.B. Gao, Messenger RNAs in dendrites: localization, stability, and implications for neuronal function, BioEssays 20 (1998) 70-78.

[12] J.P. Girard, T.A. Springer, Modulation of endothelial cells adhesion by hevin, an acidic protein associated with high endothelial venules, J. Biol. Chem. 271 (1996) 4511-4517.

[13] J. Grosche, V. Matyash, T. Moller, A. Verkhratsky, A. Reichenbach, H. Kettenmann, Microdomains for neuron-glia interaction: parallel fiber signaling to Bergmann glial cells, Nat. Neurosci. 2 (1999) 139-143.

[14] M.E. Hatten, Riding the glial monorail: a common mechanism for glial-guided neuronal migration in different regions of the developing brain, Trends Neurosci. 13 (1990) 179-184.

[15] K.S. Hoek, G.J. Kidd, J.H. Carson, R. Smith, hnRNP A2 selectively binds the cytoplasmic transport sequence of myelin basic protein mRNA, Biochemistry 37 (1998) 7021-7029.
[16] I.G. Johnston, T. Paladino, J.W. Gurd, I.R. Brown, Molecular cloning of SC1: a putative brain extracellular matrix glycoprotein showing partial similarity to osteonectin/BM40/SPARC, Neuron 2 (1990) $165-176$.

[17] C.F. Landry, J.B. Watson, T. Kashima, A.T. Campagnoni, Cellular influences on RNA sorting in neurons and glia: an in situ hybridization histochemical study, Mol. Brain Res. 27 (1994) 1-11.

[18] T.F. Lane, E.H. Sage, Review: the biology of SPARC, a protein that modulates cell-matrix interactions, FASEB J. 8 (1994) 163-173.

[19] G.L. Lyford, K. Yamagata, W.E. Kaufmann, C.A. Barnes, L.K. Sanders, N.G. Copeland, D.J. Gilbert, N.A. Jenkins, A.A. Lanahan, P.F. Worley, Arc, a growth factor and activity-regulated gene, encodes a novel cytoskeleton-associated protein that is enriched in neuronal dendrites, Neuron 14 (1995) 433-445.

[20] P. Maurer, C. Hohenadl, E. Hohenester, W. Gohring, R. Timpl, J. Engel, The C-terminal portion of BM-40 (SPARC/osteonectin) is an autonomously folding and crystallisable domain that binds calcium and collagen IV, J. Mol. Biol. 253 (1995) 347-357.

[21] D.B. Mendis, S. Shahin, J.W. Gurd, I.R. Brown, Developmental expression in the rat cerebellum of SC1, a putative brain extracellular matrix glycoprotein related to SPARC, Brain Res. 633 (1994) 197-205.

[22] D.B. Mendis, S. Shahin, J.W. Gurd, I.R. Brown, SC1, a SPARC-related glycoprotein, exhibits features of an ECM component in the developing and adult brain, Brain Res. 713 (1996) 53-63.

[23] E. Mohr, Subcellular RNA compartmentalization, Prog. Neurobiol. 57 (1999) 507-525.

[24] T. Muller, H. Kettenmann, Physiology of Bergmann glial cells, Int. Rev. Neurobiol. 38 (1995) 341-359.

[25] M. Nedergaard, Direct signalling from astrocytes to neurons in cultures of mammalian brain cells, Science 263 (1994) 1768-1771.

[26] E.A. Newman, K.R. Zahs, Modulation of neuronal activity by glial cells in the retina, J. Neurosci. 18 (1998) 4022-4028.

[27] Y. Oleynikov, R.H. Singer, RNA localization: different zipcodes, same postman?, Trends Cell Biol. 8 (1998) 381-383.

[28] A. Ortega, N. Eshhar, V.I. Teichberg, Properties of kainate receptor/channels on cultured Bergmann glia, Neuroscience 41 (1991) 335-349.

[29] S.L. Palay, V. Chan-Palay, Cerebellar Cortex, Springer-Verlag, Berlin, 1974

[30] P. Rakic, Neuron-glial relationship during granule cell migration in developing cerebellar cortex. A Golgi and electron microscopic study in Macacus rhesus, J. Comp. Neurol. 141 (1971) 283-312.

[31] J.R. Sanes, Review: extracellular matrix molecules that affect neural development, Annu. Rev. Neurosci. 12 (1989) 491-516.

[32] G. Shaw, K. Weber, The structure and development of the rat retina: an immunofluorescence microscopical study using antibodies specific for intermediate filament proteins, Eur. J. Cell Biol. 30 (1983) 219-239.

[33] G. Shaw, K. Weber, The intermediate filament complement of the retina: a comparison between different mammalian species, Eur. J. Cell Biol. 33 (1984) 95-104.

[34] T. Shiga, M. Ichikawa, Y. Hirata, Spatial and temporal pattern of postnatal proliferation of Bergmann glial cells in rat cerebellum: an autoradiographic study, Anat. Embryol. (Berlin) 167 (1983) 203211.

[35] O. Steward, Review: targeting of mRNAs to subsynaptic microdomains in dendrites, Curr. Opin. Neurobiol. 5 (1995) 55-61.

[36] O. Steward, Review: mRNA localization in neurons: a multipurpose mechanism?, Neuron 18 (1997) 9-12.

[37] B.D. Trapp, T. Moench, M. Pulley, E. Barbosa, G. Tennekoon, J. Griffin, Spatial segregation of mRNA encoding myelin-specific proteins, Proc. Natl. Acad. Sci. U.S.A. 84 (1987) 7773-7777.

[38] K. Venstrom, L.F. Reichardt, Review: role of extracellular matrix molecules and their receptors in the nervous system, FASEB J. 7 (1993) 996-1003. 